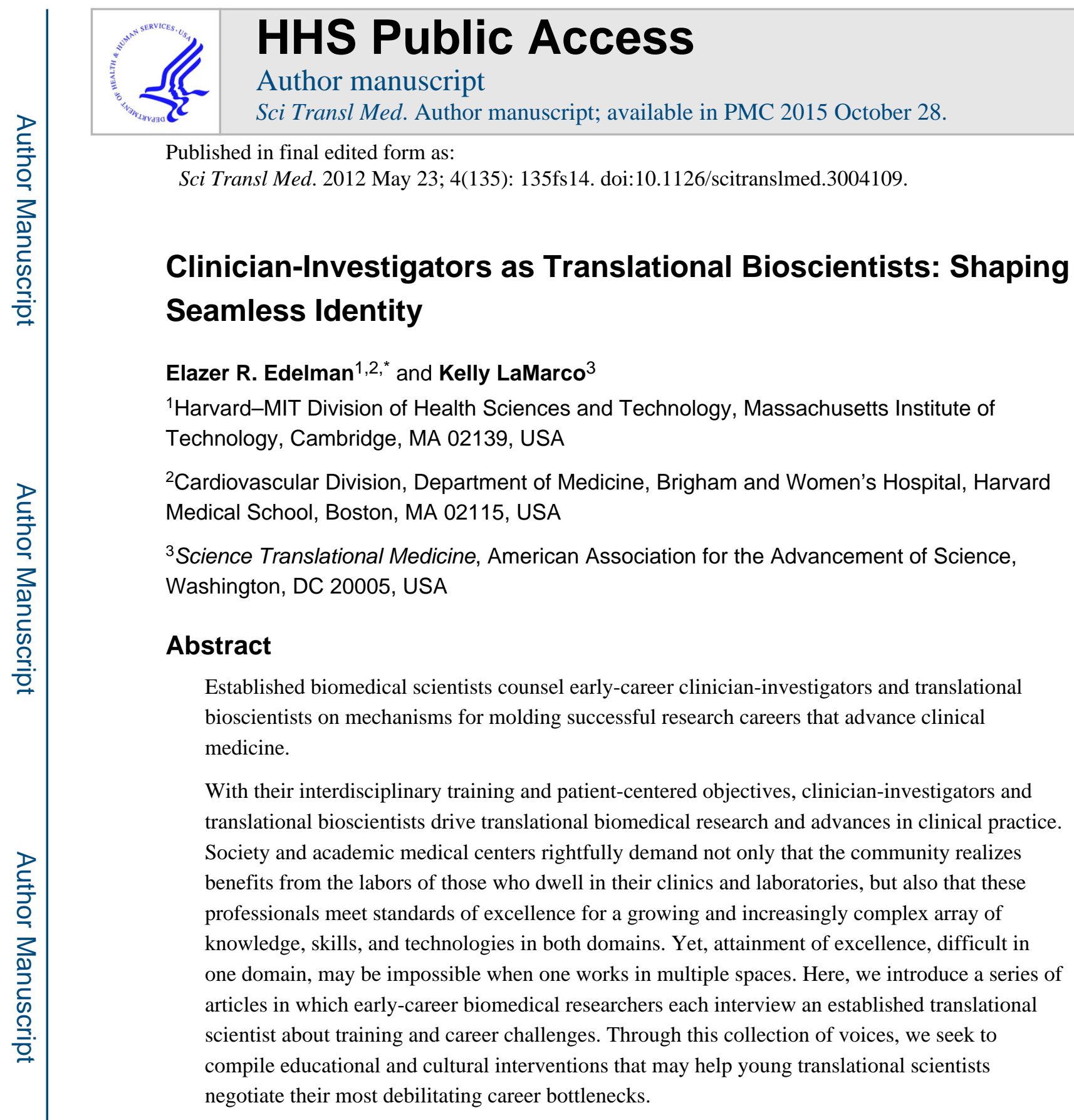

\title{
MISSION IMPOSSIBLE?
}

For some professionals, translation of scientific knowledge requires clinical exposure, and for a time, there was minimal distinction between the clinician-investigator and the translational bioscientist. But science and medicine have evolved, and the veracity of this generalization must be reevaluated. Translational bioscientists acknowledge that the privilege of performing clinically motivated scientific investigation ultimately requires clinical fulfillment. Clinician-investigators - who both care for patients and pursue biomedical research — take this mantra to an extreme, holding that an effective clinical

*Corresponding author. ere@mit.edu. 
presence requires complementary investigative research and that research performed by medical practitioners necessitates clinical benefit in return.

This integrated mission has been most effectively fulfilled when a physician who cares for patients and conducts laboratory research performs both vocations as a single persona: that of the clinician-investigator (Fig. 1). However, the current state of biomedicine as well as economic and social conditions force us to explore whether it is possible to assume a seamless identity that embraces excellence in both clinical and investigative sciences in the pursuit of improved human health and whether only one path and one professional identity can promote the translation of scientific discoveries to clinical advances.

\section{A SEAMLESS BUT ELUSIVE IDENTITY}

Much has been written about the optimal career balance of the successful academic clinician-investigator. Some argue that the appropriate balance can be represented by a three-legged stool, with the legs representing clinical medicine, biomedical research, and education/mentoring; the stool is stable when the lengths of the legs are matched, indicating equal excellence in all three domains. Those who hold this view claim that the simultaneous pursuit of excellence in all three areas drives achievement in each domain to a higher level than could be reached if one focused primarily on a single leg of the stool. Others argue the translational bioscientist has similar responsibilities - obliged to teach, investigate and assure clinical impact, propelling concepts into communal good.

However, questions surround the viability of the clinician-investigator career path: Can medical practitioners who simultaneously seek to become translational bioscientists function effectively as full-fledged clinician-investigators? The deluge of biomedical data, increasingly complex new technologies, and growing bases of specialty knowledge propel research and discovery but also make it difficult, if not impossible, for a single person to master all three legs of the stool in equal measure. Some argue that the legs grow asymmetrically, creating a precarious imbalance that makes sitting stably atop the stool impossible to achieve, unlikely to promote translation, and dangerous for patients and careers.

How has this meta-stable platform changed the careers of newly minted clinicianinvestigators? If the intangible concerns on junior clinician-investigators center about the ability to meet the concomitant demands of two worlds, the tangible, quantifiable, and growing stresses forced by the intangibles are manifest as increases in the length of training and time to independence. In this era of integrated mechanistic medicine, long training times are required to become clinically proficient and to remain clinically adept, and in a similar vein to complete scientific rites of passage. A 2002-2003 study showed a median registered time to a Ph.D. degree in the life sciences of 7 years-up almost $20 \%$ from 5.9 years in 1978 (1) — and MD-PhD trainees who graduated between 1998 and 2007 took 7.8 years to complete both degrees (2). Residency training for MDs and MD-PhDs lasts $\sim 3$ years, depending on the discipline, and expands with specialization, adding up to an additional 4 years. Presumably, those who return to research after intense isolated clinical immersion 
must pursue postdoctoral scientific training to master new methods and knowledge to be competitive in the biomedical research arena.

After completion of postgraduate studies, clinician-investigators tread a lengthier and more tenuous path toward their first assistant professorships and first major independent grants than ever before. In 1980, average ages at time of appointment to assistant professorship in the biomedical sciences were 33.5, 33.5, and 34.8 years for PhDs, MDs, and MD-PhDs, respectively, rising in 27 years to $38.5,37$, and 39.9 years, respectively. Similarly, in 1980 the average ages of PhDs, MDs, and MD-PhDs in the biomedical sciences when they received their first U.S. National Institutes of Health (NIH) RO1 grants were 35.7, 37.7, and 36.1 years, respectively, rising to 41.7, 44.2, and 42.3 years, respectively, in 2007 (3). All took substantially longer to gain faculty slots and the R01 status of independence; MDs became faculty members sooner than those with $\mathrm{PhDs}$ but became independent later. Thus, it appears that postdoctoral training nearly doubled in duration over this time period but was virtually required before a clinician-investigator could achieve some level of professional security. Is it reasonable and sustainable to require more than two decades of training after college matriculation before a clinician-investigator can take charge of his or her own professional fate?

To function as clinician-investigators, young professionals must juggle the need for training to excellence in clinical medicine with the need to spend more time doing science, just as the pace of research support has fallen well behind the rise in expense. But can a clinicianinvestigator ever be as competent a physician as $\mathrm{s} /$ he could be if $\mathrm{s} / \mathrm{he}$ cared for patients alone, or as competitive a scientist as s/he could be if $\mathrm{s} / \mathrm{he}$ had no clinical responsibilities to balance? In a recent survey (2), career data were collected from nearly 6000 current and former trainees in $24 \mathrm{NIH}$-funded MD-PhD training programs. Among respondents who completed their MD-PhD programs and worked in academic medical centers, $82 \%$ were engaged in research, but close to two thirds of these noted that they spent only $50 \%$ of their time in the lab. Just $39 \%$ devoted more than $75 \%$ of their efforts to research.

There are few data that provide insight into what percentage of biomedical scientists truly embody the single persona of the clinician-investigator in their careers. Receipt and renewal of RO1 grants are metrics for assessing research proficiency, but it is unclear whether the focus is on basic research or science driven by unmet clinical needs. Comparable metrics for clinical proficiency are difficult to quantify. Data exist on the career desires and fields of interest of new MDs and MD-PhDs when they enter the work force (4) but not on whether these professionals ultimately practice medicine while conducting patient-driven research with the goal of improving clinical medicine. This is information we need going forward if we are to establish a prognosis for the clinician-investigator.

\section{ORCHESTRATING AN INTERVENTION}

In the context of the heated debate on optimal career balance, we seek to answer basic sets of questions: What do modern careers in academic medicine and the translational biosciences look like, and how can they be attained? Can the timeline of training that now entails full clinical and full research exposure be shortened and curriculum streamlined, or is 
the mastery of multiple trades by its very nature strenuous and time-consuming? Is combined excellence in both clinical and investigative sciences required for the advancement of translational medicine? Last, how can one define, in a rigorous manner, how well the current training system is working and the potential impact of interventionsthat is, alterations in the system of academic personal development that affect curriculum or training protocols? Answers to such questions require delineation of the conflicts and bottlenecks faced by current early-career biomedical scientists and an analysis of possible curricular and cultural interventions that may help circumvent obstacles to a successful and rewarding career as a clinician-investigator.

In the new article series, we attempt to define such educational interventions using an approach that is akin to the delineation process for any clinical syndrome and the evaluation protocol for any potential intervention. In medicine, case studies of individual patients identify potential association of signs and symptoms with a disease process. Larger studies that examine a significant and scientifically selected segment of the population can determine the incidence of disease. Increasingly broad sampling can refine the emerging list of signs and symptoms, offer a mechanism of action to explain the disease, create mechanistic and thematic models to group patients, and help suggest potential interventions. These interventions, however, can only be assessed in full-fledged clinical trials.

Our strategy is to apply the same paradigm to define and evaluate educational interventions. We are now at the point of accumulating case reports to define commonalities and derive mechanistic insights into critical issues that will then suggest interventions. To tap into the knowledge one achieves through experience, we start with case studies of early-career clinician-investigators and translational bioscientists who discuss a specific set of challenges with senior clinical and translational scientists. The questions raised reflect the specific needs of the emerging faculty member, and the answers reflect the observations and guidance of the established faculty member-each based on their respective experiences. The collation of these cases will help to determine whether a syndrome can be defined and a model created that circumscribes the issues at hand and suggests interventions. These suggested changes might then be evaluated more formally in a trial that has specific goals and metrics to determine the health of the system and impact of intervention.

The first interview, in this issue of Science Translational Medicine (5), pinpoints two essential ingredients for a high-impact career as a clinician-investigator: (i) contact with patients, which highlights unmet medical needs, and (ii) a willingness to change fields and pursue risky research with uncertain outcomes. One possible intervention, then, would be for institutions and funders to create an environment in which taking risks is encouraged and rewarded. A second might be for clinician-investigator training programs to institute a selection process that favors versatile and daring students who thirst for a deeper understanding of human physiology and pathophysiology.

In future issues, we will address other aspects of the changing landscape confronting young investigators who are interested in clinically motivated translational research. We will present work and life issues of translational scientists who have trained clinically without receiving a physician's degree, those whose work is clinically motivated but not clinically 
involved, and those who gained exposure to translational science late in a medical career. We look forward to an extended discussion of critical career bottlenecks and transformative solutions that shape biomedical scientists who are capable of advancing translation.

\section{References and Notes}

1. Hoffer, TB.; Welch, V, Jr. Infobrief: Time to degree of US research doctorate recipients. National Science Foundation; Mar. 2006

2. Brass LF, Akabas MH, Burnley LD, Engman DM, Wiley CA, Andersen OS. Are MD-PhD programs meeting their goals? An analysis of career choices made by graduates of $24 \mathrm{MD}-\mathrm{PhD}$ programs. Acad Med. 2010; 85:692-701. Medline. [PubMed: 20186033]

3. NIH Office of Extramural Research, Data on newinvestigators. NIH Workforce Discussions; PowerPoint presentation. Sep. 2007 available at http:/grants.nih.gov/archive/grants/ new_investigators/index.htm

4. Andriole DA, Whelan AJ, Jeffe DB. Characteristics and career intentions of the emerging $\mathrm{MD} / \mathrm{PhD}$ workforce. JAMA. 2008; 300:1165-1173. Medline. 10.1001/jama.300.10.1165 [PubMed: 18780845]

5. Henrickson S. Risk and return for the clinician-investigator. Sci Transl Med. 2012; 4:135cm6.

6. Image credit: This image is a faithful photographic reproduction of an original two-dimensional work of art and is in the public domain. The work of art itself is in the public domain because its copyright has expired. This applies to those countries (including the United States) with a copyright term of life of the author plus 70 years. http://en.wikipedia.org/wiki/

Klimt_University_of_Vienna_Ceiling_Paintings 


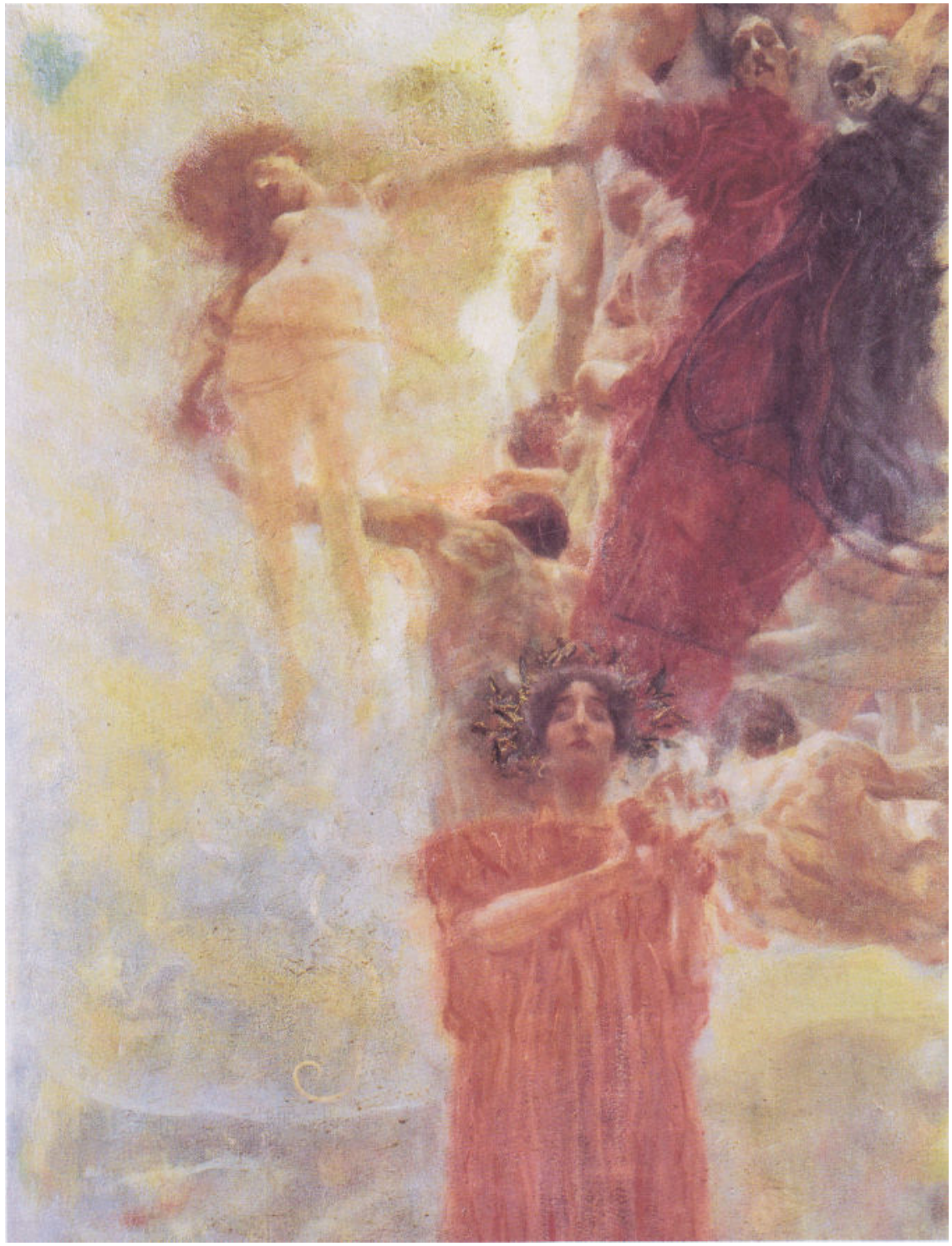

Fig. 1. Multiple missions, one persona

Despite their kaleidoscopic career paths, clinician-investigators must adopt a single, integrated persona to effectively care for patients with complex diseases and conduct translational research with the potential to transform clinical medicine. [Medicine by Austrian artist Gustav Klimt (1862-1918)]

CREDIT: See (6). 